\title{
Nanowire formation by gold nano-fragment coalescence on quantized vortices in He II
}

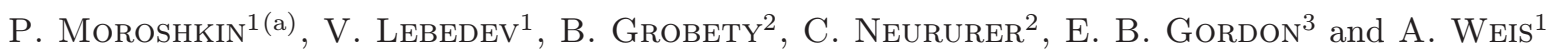 \\ ${ }^{1}$ Department of Physics, University of Fribourg - Chemin du Musée 3, 1700 Fribourg, Switzerland \\ ${ }^{2}$ Department of Geoscience, University of Fribourg - Chemin du Musée 6, 1700 Fribourg, Switzerland \\ ${ }^{3}$ Institute of Problems of Chemical Physics RAS - Acad. Semenov av. 1, 142432 Chernogolovka, Russia
}

\begin{abstract}
PACS 47.37.+q - Hydrodynamic aspects of superfluidity; quantum fluids
PACS 67.25.dk $-{ }^{4} \mathrm{He}$ : Vortices and turbulence

PACS $61.46 . \mathrm{Km}$ - Structure of nanowires and nanorods (long, free or loosely attached, quantum wires and quantum rods, but not gate-isolated embedded quantum wires)
\end{abstract}

\begin{abstract}
We demonstrate that laser ablation of a gold target immersed in superfluid and normal fluid helium leads to the formation of elongated gold nano-fragments. In the superfluid phase these nano-fragments aggregate into filaments with extremely large aspect ratios displaying metallic electric conductivity. We attribute this unusual structure to the coalescence of gold particles trapped on quantized vortices. Our observations suggest new ways to visualize the structure of quantized vortex bundles and a new approach for producing centimeter-long metal nanowires.
\end{abstract}

Superfluid ${ }^{4} \mathrm{He}$ (HeII) exhibits the outstanding macroscopic quantum property of superfluidity when cooled below $2.172 \mathrm{~K}$. Associated with this property is the appearance of one-dimensional structures around which the fluid motion has a circulation that is quantized in units of $h / m_{\mathrm{He}}$. Such vortex lines have been studied in detail for more than 50 years as reported in the comprehensive review by Donnelly [1]. More recently the flow of superfluid and normal fluid helium has received renewed attention in the context of quantum turbulence $[2,3]$.

Because of the strong pressure gradient in the vicinity of their core, quantized vortices can bind foreign particles. This fact has been used for the visualization of vortex bundles by the electrostatic extraction and projection of vortex-attached electrons onto a phosphor screen [4]. The pressure gradients are strong enough to attract also heavier particles, such as ${ }^{3} \mathrm{He}$ or other guest atoms and clusters. Because of the one-dimensional structure of the vortex line one expects [5] the formation of monoatomic chains or high-aspect-ratio filaments following the coalescence of particles on the vortex core. The formation of macroscopic filament-like structures by impurity particles suspended in superfluid helium was indeed reported by several authors [6-9] as well as the lining-up of micronsized solid hydrogen clusters injected into normal fluid $\mathrm{He}$,

\footnotetext{
(a) E-mail: peter.moroshkin@unifr.ch
}

followed by a transition to the superfluid phase [10,11]. No such unidimensional structures appear in normal fluid helium. All previous studies were limited to visual observations in the He bath only, since the filament materials were either hydrogen or alkali metals which oxidize in contact with air. The experimental results presented below suggest a new approach to this problem. By doping liquid helium with a chemically inert material that keeps its shape and properties after the helium evaporation, we were able to form solid filaments and analyze them by means of optical and electron microscopy. In doped HeII we observe the production of centimeter-long gold filaments, whose analysis reveals a very rich substructure at the nanometer scale and the formation of metallic binding. On the other hand, when the doping is done in normal fluid helium we obtain only chaotically oriented gold nanonetworks which (because of their small size) escaped observation in previous studies. Our observations open new ways for visualizing the patterns of the superfluid and normal fluid helium motion (turbulence) and for producing nanowires with extreme aspect ratios.

The experiments were performed in the experimental setup used in our previous spectroscopic studies of alkali atoms and molecules in solid ${ }^{4} \mathrm{He}$ matrices [12]. Atoms are introduced into superfluid or normal fluid helium by laser ablation with pulsed frequency-doubled Nd:YAG laser radiation focused on a gold target at the bottom of 


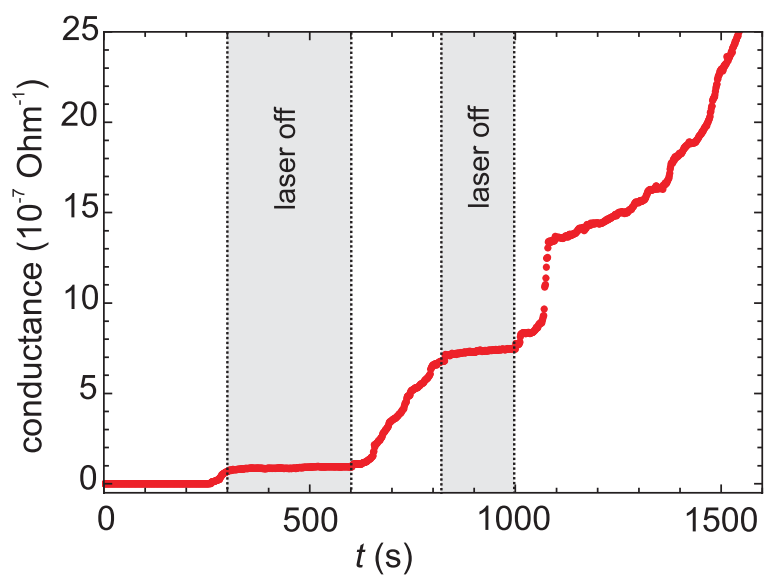

Fig. 1: (Colour on-line) Electric conductance of gold nanowires in superfluid helium ( $T=1.5 \mathrm{~K}, p=22$ bar) during laser ablation, initiated at time $t=0$.

a copper cell filled with normal fluid or superfluid helium, and immersed in a He bath whose temperature could be varied from 1.5 to $2.7 \mathrm{~K}$. The sample can be visually inspected through four side windows, with a top window used for admitting the ablation laser beam.

In order to detect the appearance of conductive gold wires, the cell contained two vertical flat electrodes made from polished copper foil, spaced by $4 \mathrm{~cm}$. During laser ablation the electrical resistance between the electrodes and between the electrodes and the cell walls (kept at ground potential) was monitored. All wires produced during laser ablation in superfluid helium eventually fall onto $0.5 \mathrm{~mm}$ thick microscope slides and carbon-coated copper grids arranged at the bottom of the cell. In this way the wires could be removed after warming up the cryostat and investigated in detail by means of optical, scanning (SEM) and transmission (TEM) electron microscopy. For the SEM analysis the gold microstructures were stabilized on the glass slides by covering them with a $1.5 \mathrm{~nm}$ thick carbon film; for the TEM study we use the material collected directly on the carbon-coated copper grids. The chemical composition of the nanofibers was analyzed by energy-dispersive spectroscopy (EDS, EDAX attached to the SEM). X-ray spectra recorded at an acceleration voltage of $10 \mathrm{kV}$ revealed a small copper contamination. The latter probably comes from the cell walls or the target holder occasionally hit by the ablation laser. All other impurities present in the cell or in the $\mathrm{He}$ gas at the temperature of the experiment were frozen at the cell walls and did not contaminate the sample.

A first indication for the appearance of nanowires was obtained by measuring the electric conductivity of golddoped superfluid He. A typical measured time dependence of the electric conductance between an electrode and the cell wall is shown in fig. 1 . The conductance was less than $100 \mathrm{M} \Omega^{-1}$ before firing the first laser pulse and starts to grow approximately 250 seconds after switching on the ablation laser (firing at a rate of $10 \mathrm{~Hz}$ ). The unaided

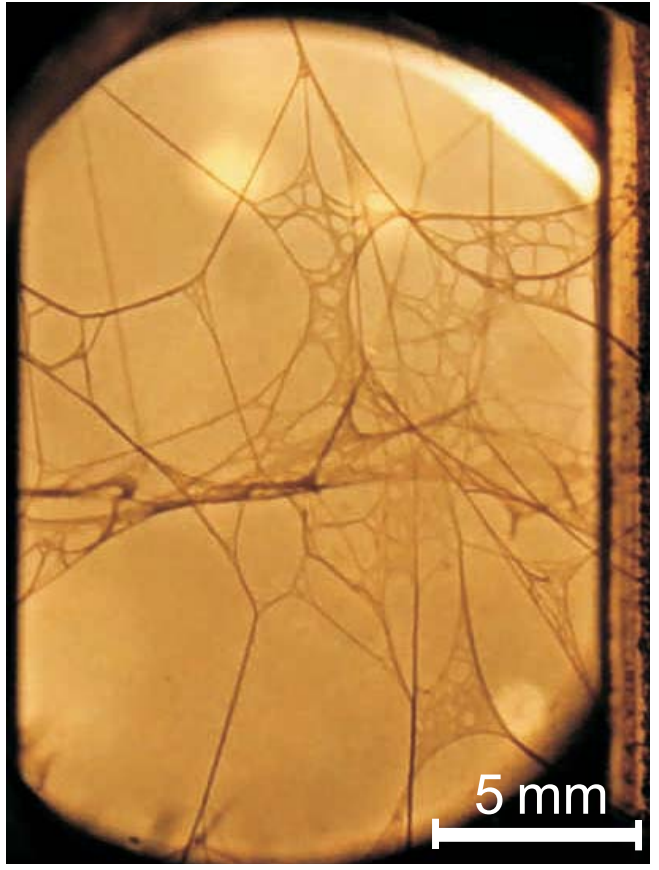

Fig. 2: (Colour on-line) Photograph taken through the $20 \mathrm{~mm}$ diameter side window of the pressure cell at the end of a laser ablation series with a copper target in superfluid helium.

eye cannot observe any objects in the helium during that phase. Figure 1 also shows that the conductance increase comes to a halt within a few seconds after switching off the laser. We have applied several series of laser ablation pulses, each about one hour long $(T=1.5-2.0 \mathrm{~K}$, $p=2-25$ bar $)$. At the end of every series each electrode was found to be electrically connected to the cell walls with a resistance $R_{f}$ of $30-200 \mathrm{k} \Omega$. Assuming that the filaments have no contact resistance with the electrodes and the cell walls, that the filament(s) has (have) a length of $1 \mathrm{~cm}$ (the shortest electrode-wall distance), and that the filament resistivity is that of bulk gold, the resistance $R_{f}$ corresponds to a filament cross-section of $10-70 \mathrm{~nm}^{2}$. In fact, at the end of the ablation process the gold filaments had a much larger thickness and could easily be seen by unmagnified visual inspection. It is therefore likely that the measured resistance represents mainly contact resistance(s).

A photograph taken through a side window of the pressure cell at the end of the experimental run is shown in fig. 2. One clearly sees a spider-web-like structure made of several thick wires. The characteristic length of each wire is comparable to the cell dimensions. Changing the helium pressure in the cell and, in particular the $\mathrm{He}$ solidification at 26 bar breaks the thinner wires or detaches them from the cell walls and the electrodes, as can be monitored by conductance measurements. However, the thicker wires in fig. 2 do survive several helium solidification-melting cycles and are detached/destroyed only by several pulsed admissions of $\mathrm{He}$ gas into the 

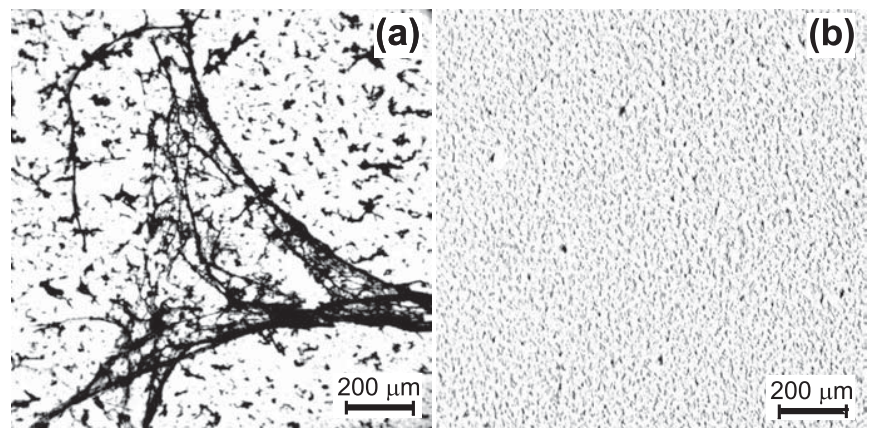

Fig. 3: Optical microscope images of gold filaments and particles deposited during the laser ablation in superfluid $\mathrm{He}$ at $1.5 \mathrm{~K}(\mathrm{a})$ and in normal fluid $\mathrm{He}$ at $2.7 \mathrm{~K}$ (b).
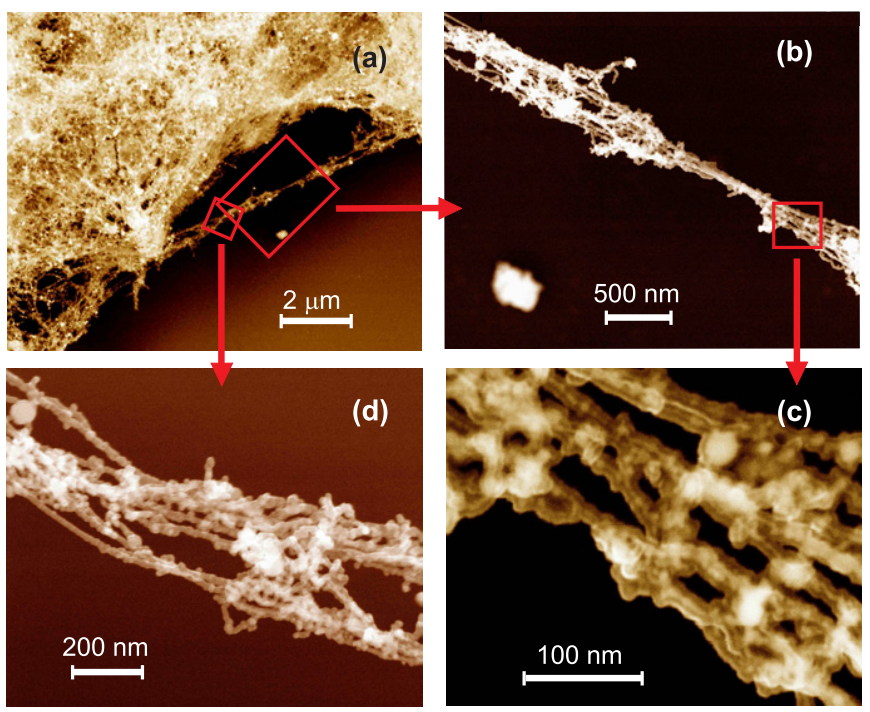

Fig. 4: (Colour on-line) SEM images of gold nanowires produced in superfluid He at $1.5 \mathrm{~K}$, removed from the pressure cell and stabilized on a glass surface by a carbon film.

cell, which produce an abrupt pressure and temperature increase. We have obtained very similar web structures in a series of ablation experiments with gold, copper, barium, cesium, and rubidium. In particular, the web shown in fig. 2 is made of copper. The electron microscopy studies described below were carried out only on gold samples. One can expect that the nanostructures formed by different metals are very similar.

The filaments collected after the warming of the cryostat were analyzed by optical, secondary electron (SEM) and transmission electron (TEM) microscopy. A typical lowresolution optical microscope image of a thick wire is shown in fig. 3(a). Already at this low magnification it becomes apparent that the wires are in fact bundles formed by a large number of much thinner filaments. Typical SEM images recorded with electron energies of $10 \mathrm{keV}$ are shown in fig. 4. In the lowest-magnification image (fig. 4(a)) a very dense web-like structure is visible that is made from a large number of fibers containing
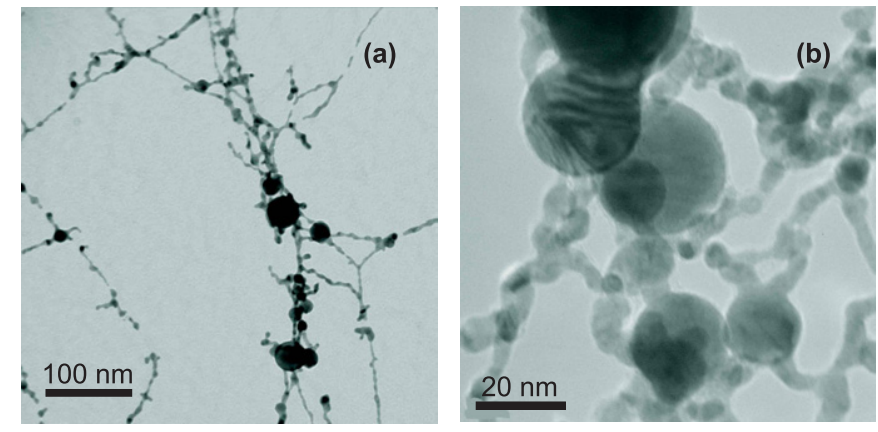

Fig. 5: Typical TEM BF images of gold nanowires and spherical nanoparticles deposited on a carbon-coated copper grid in superfluid helium.
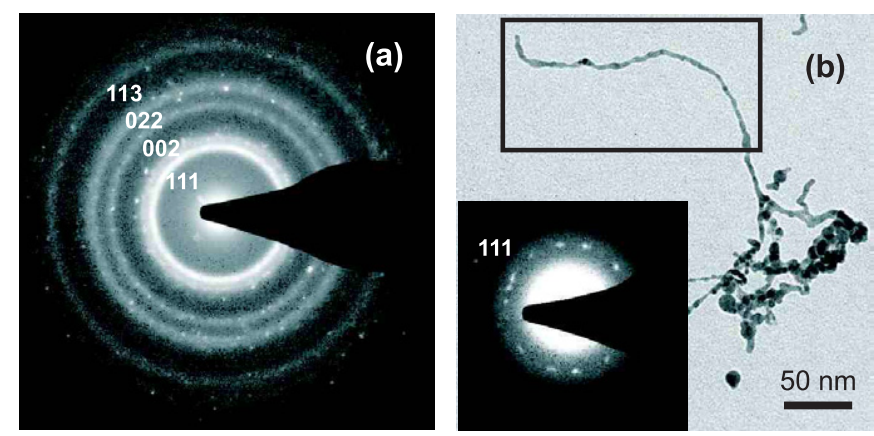

Fig. 6: (a) SAED pattern of a gold nanowire web produced in superfluid helium; (b) individual gold nanowire (box) with corresponding SAED pattern (inset).

inclusions of spherical particles with diameters of some $100 \mathrm{~nm}$. Figures 4(b)-(d) show higher-resolution SEM images of different parts of this web structure. It is difficult to infer the diameter of individual fibers from these SEM images because of the carbon coating that was used to fix the sample onto the substrate. However, the nanowires are seen to be entangled and to form thicker ropes, as shown in fig. 4(b). The thickness of those ropes may be as large as $100 \mu \mathrm{m}$ (fig. 2) and they become visible to the naked eye. The characteristic length of an individual nanowire is larger than the width of the scan in fig. 4(b), i.e., $>3 \mu \mathrm{m}$, whereas a rope typically has a length comparable to the cell dimensions, i.e., $\simeq 6 \mathrm{~cm}$. It is thus conceivable that the length of a nanowire - when produced under more controlled conditions - can reach several centimeters and will be limited only by the container size.

Typical bright-field (BF) TEM images are shown in fig. 5. Diameters of individual wires range from approximately 1.5 to $6 \mathrm{~nm}$. The thinnest parts of the wires do not survive electron bombardment in the microscope. They are severed, leaving behind typical gaps along the wire (fig. 5(a)). Spherical gold nanoparticles are irregularly distributed along the wires. Selected-area electron diffraction (SAED) patterns taken from a web-like part of the sample can be indexed for the fcc (face-centered cubic) crystalline structure of gold (fig. 6(a)). The diffraction 

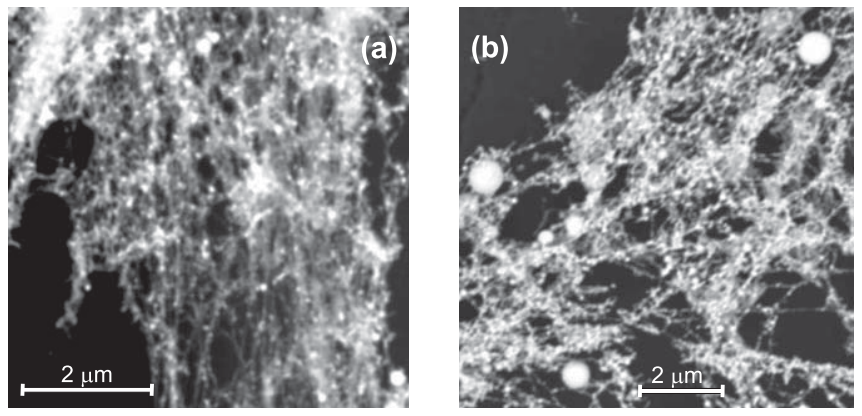

Fig. 7: Comparison of nano networks produced in superfluid helium at $1.5 \mathrm{~K}$ (a) and in normal fluid helium at $2.7 \mathrm{~K}$ (b).

rings are broad due to the very small size of the wires. SAED patterns taken from individual wires (fig. 6(b)) indicate that the crystallographic orientation may vary along the wire. It is well known that the crystalline structure of gold nanowires changes progressively with decreasing radius. For a wire with a $2 \mathrm{~nm}$ diameter, Kondo and Takayanagi have deduced [13] that the core has a fcc structure, whereas the surface layer is restructured in a hexagonal symmetry. Wires smaller than $\approx 1.5 \mathrm{~nm}$ have a two- or three-layer shell structure [14]. It is likely that such nonfcc structures also occur in the present experiment in the thinnest sections of the wires.

In order to elucidate the role of quantized vortices in the nanowire formation, we have carried out the same experimental protocol in normal fluid helium at $2.7 \mathrm{~K}$. No quantized vortices exist under those conditions, however, it is likely that the thermal convection from the heat deposited by the ablation laser creates a strongly turbulent flow of liquid helium in the vicinity of the ablation target. In agreement with earlier experiments, no filament formation could be observed in normal fluid helium even after several hours of laser ablation. A typical optical microscope image of deposited material is shown in fig. 3(b) and supports the visual observations. The laser ablation in the normal fluid produces gold particles that are smaller than those obtained in the superfluid (fig. 3(a)) and more uniformly distributed on the substrate. However, the electron-microscopy analysis of the deposited material reveals a very interesting picture. A characteristic SEM image is shown in fig. 7(b). Rather than nanowires organized in thick ropes one observes a large number of relatively short elongated nanoscopic fragments randomly scattered on the substrate and forming a nano-network. For a comparison, another SEM image of the material deposited in superfluid helium is shown in fig. 7(a). The characteristic thickness of individual nano-fragments produced in normal fluid $\mathrm{He}$ is quite similar to that of a nanowire produced in the superfluid. In fact, at a submicrometer scale one hardly can distinguish the structures produced in the two phases.

Laser ablation of metals in liquid helium produces a large number of metallic clusters $[7,15,16]$ that dominate over the number of isolated atoms. We have determined earlier the diameters of metal clusters to lie in the range of 10-100 nm [16], comparable in size to the spherical gold nanoparticles shown in figs. 4 and 5 .

The nanoparticles created by laser ablation can be dissociated into atoms and dimers by a second sputtering laser, a method that we have used for our spectroscopic studies of atomic and molecular impurities in liquid and solid $\mathrm{He}[12]$. We have confirmed the presence of $\mathrm{Au}$ atoms and $\mathrm{Au}_{2}$ molecules in our experimental cell via their laser-induced fluorescence spectra [17]. The typical diameters $(\approx 5 \mathrm{~nm})$ of the gold nanowires make us assume that they are formed from individual $\mathrm{Au}$ atoms and/or very small clusters extracted by the ablation laser either from the target surface or from larger nanoparticles in the superfluid matrix. The latter process should be particularly efficient since the wavelength of the ablation laser $(532 \mathrm{~nm})$ is very close to the plasmon resonance of gold nanoparticles [18].

Nano-fragments of comparable sizes are produced in large numbers both in the normal and superfluid phase of liquid helium. We therefore conclude that the microscopic mechanism of the nanowire formation is not specific for the superfluid phase, as it has been assumed before [5,9-11].

Metal atoms in condensed He reside in nanometer-sized cavities, so-called atomic bubbles [12]. He atoms with their closed electronic shells are expelled from the volume occupied by valence electrons of the metal atom because of the Pauli principle. Similar bubbles are formed by free electrons in superfluid He. The ablated metal atoms, or rather the associated atomic bubbles can move together with the flow of liquid helium. In superfluid helium they can be trapped by a quantized vortex because of the radial pressure gradient

$$
\frac{\mathrm{d} p}{\mathrm{~d} r}=\frac{\rho_{s} v_{s}(r)^{2}}{r}=\frac{\rho_{s} \Gamma^{2}}{4 \pi^{2} r^{3}},
$$

produced by the motion of the superfluid around the vortex core $[1,19,20]$, where $\rho_{s}$ and $v_{s}(r)$ are the superfluid density and velocity, and $\Gamma=h / m_{\mathrm{He}}$ is the quantum of circulation.

We suggest that in the normal fluid phase a similar mechanism may exist due to the presence of eddy currents in the turbulent flow around the ablation target that is strongly heated by the laser. The lifetimes of these eddies, their stability and their typical lengths are much smaller than those of quantized vortices in the superfluid. Nanofragments thus cannot aggregate into high-aspect-ratio ropes and macroscopic filaments. More experimental and theoretical work is needed in order to clarify the nanowire formation mechanism. We hope that our experimental findings will attract the attention of theoreticians and can advance the understanding of the motion of quantum fluids and in particular of quantum turbulence.

In conclusion, we have demonstrated that laser ablation from metal targets in superfluid and normal fluid 
${ }^{4} \mathrm{He}$ results in the formation of spherical and elongated nanoparticles with very large aspect ratios. In the superfluid phase these nanowires reach particularly large lengths and become entangled to form macroscopically large filaments. We suggest that the nanowires are formed by the pressure-gradient-driven coalescence of the clusters and nano-fragments produced by the ablation onto quantized vortex lines and/or normal fluid eddies.

This work was supported by grant No. 200020-119786 of the Swiss National Science Foundation. EBG thanks the University of Fribourg for hospitality and the Light\&Matter (LiMat) collaboration for financial support. We acknowledge stimulating discussions with J. P. Toennies and C. A. Stafford.

\section{REFERENCES}

[1] Donnelly R. J., Quantized Vortices in Helium II (Cambridge University Press, Cambridge) 1991.

[2] Vinen W. F. and Niemela J. J., J. Low Temp. Phys., 128 (2002) 167.

[3] Niemela J. J. and Sreenivasan K. R., J. Low Temp. Phys., 143 (2006) 163.

[4] Yarmchuk E. J., Gordon M. J. V. and Packard R. E., Phys. Rev. Lett., 43 (1979) 214.
[5] Gordon E. B. and Okuda Y., Low Temp. Phys., 35 (2009) 209.

[6] Silvera I. F., Phys. Rev. B, 29 (1984) 3899.

[7] Fujisaki A., Sano K., Kinoshita T., Takahashi Y. and Yabuzaki T., Phys. Rev. Lett., 71 (1993) 1039.

[8] ARndt M., PhD Thesis, Ludwig Maximilians Universität München, Max-Planck-Institut für Quantenoptik, Garching (MPQ-Report 197) 1995.

[9] Gordon E. B., Nishida R., Nomura R. and Okuda Y., J. Exp. Theor. Phys. Lett., 85 (2007) 581.

[10] Bewley G. P., Lathrop D. and Sreenivasan K. R., Nature, 441 (2006) 588.

[11] Paoletti M. S., Fiorito R. B., Sreenivasan K. R. and Lathrop D. P., J. Phys. Soc. Jpn., 77 (2008) 111007.

[12] Moroshinin P., Hofer A. and Weis A., Phys. Rep., 469 (2008) 1.

[13] Kondo Y. and Takayanagi K., Phys. Rev. Lett., 79 (1997) 3455.

[14] Kondo Y. and Takayanagi K., Science, 289 (2000) 606.

[15] Beijersbergen J. H. M., Hui Q. and Takami M., Phys. Lett. A, 181 (1993) 393.

[16] Moroshinin P., Hofer A., Ulzega S. and Weis A., Nat. Phys., 3 (2007) 786.

[17] Moroshinin P., Lebedev V., Gordon E. B., Toennies P. and WEIS A., in preparation (2010).

[18] Mafune F., Kohno J., Takeda Y. and Kondow T., J. Phys. Chem. B, 107 (2003) 12589.

[19] Berloff N. G. and Roberts P. H., Phys. Rev. B, 63 (2000) 024510.

[20] Kivotides D., Barenghi C. F. and Sergeev Y. A., Phys. Rev. B, 77 (2008) 014527. 